\title{
Bulk Compounding of PCL-PEO Blends for 3D Plotting of Scaffolds for Cardiovascular Tissue Engineering
}

\author{
Ragaert $\mathrm{K}^{1^{*}}$, Maeyaert $\mathbf{G}^{1}$, Martins $\mathrm{Cl}^{2}$, and Cardon $\mathbf{L}^{1}$ \\ ${ }^{1}$ Centre for Polymer and Material Technologies, Faculty of Engineering and Architecture, Ghent University, Belgium \\ ${ }^{2}$ Institute for Polymer and Composites/I3N, University of Minho, Guimaraes, Portugal
}

"Corresponding author: Kim Ragaert, Centre for Polymer and Material Technologies, Faculty of Engineering and Architecture, Ghent University, Belgium, Tel: +32-9-2422665; E-mail: kim.ragaert@ugent.be

Rec date: Sep 30, 2013, Acc date: Feb 23, 2014, Pub date: Mar 01, 2014

Copyright: (c) 2014 Ragaert K, et al. This is an open-access article distributed under the terms of the Creative Commons Attribution License, which permits unrestricted use, distribution, and reproduction in any medium, provided the original author and source are credited.

\begin{abstract}
3D plotting of micro-extruded filaments is a promising method for the additive manufacturing of porous scaffolds (for tissue engineering) in thermoplastic polymers. A much investigated polymer for degradable scaffolds is poly- $\varepsilon$ caprolactone (PCL). One of the remaining issues with the material is its inherent hydrophilicity, which leads to nonspecific protein adsorption. Specifically for cardiovascular applications, it has also been found that PCL is insufficiently flexible to mimic the mechanic-elastic behavior of the natural tissue. Earlier research has shown that blending with low molecular weight poly-ethylene-oxide (PEO) may offer an improvement in terms of both hydrophilicity and flexibility. Until now, solution-based blending has been used as a manufacturing method for these blends, since PCL and PEO are largely immiscible in the melt. This, however, is tedious work which yields only a few grams of material at a time. Therefore, in the current research, a method has been developed for the bulk compounding of PCL-PEO blends, using a twin-screw extruder. The manufactured blends were evaluated for composition, dispersion of the PEO and crystalline morphology. It was found that by re-compounding the blends after the first extrusion step, a good dispersion of the PEO within the PCL matrix was achieved.
\end{abstract}

Keywords: Twin screw extrusion; PCL; PEO; Blends; Morphology; Scaffolds

\section{Introduction}

More and more, biodegradable polymers find an application within the domain of tissue engineering, where they are used as materials for the production of degradable scaffolds. These are support structures for the culture of the patient's harvested cells in an in vitro environment. While this neo-tissue grows, the scaffold slowly degrades into nontoxic components, eventually leaving only the new, functional and healthy tissue behind. This final construct can be implanted into the patient and will not solicit any rejection, because the cells used are the patient's own.

For cardiovascular application and more specifically leaflets for heart valve replacement, the elastic-mechanical properties of these scaffolds are just as important as their biodegradability and the nontoxicity of the material. The leaflet must be strong enough to withstand the blood pressure and at the same time be able to follow the elastic movement of a natural valve. In fact, when researching such valve scaffolds, we do not look for the strongest possible scaffold but for the highest possible compliance with the elastic-mechanical behavior of natural tissue. Adherence to such compliant behavior will provide the correct mechanical stimuli for the differentiation of the seeded cells.

The aliphatic polyester poly- $\varepsilon$-caprolactone (PCL) is quite suitable as a base material for scaffolds within this research because it is relatively flexible [1,2] and has excellent thermal stability [3], which makes it very suitable for use with a melt processing technique like the micro-extrusion for 3D plotting proposed within this work (Figure 1). PCL is considered to be compatible with both hard and soft tissues
[4-6] and will degrade slowly in the human body over a period of 24 to 36 months $[7,8]$.

The main disadvantage of PCL is the strongly hydrophobic nature of its surface [9-11], which will lead to highly undesired non-specific protein adsorption [12].

A possibly solution for this is the blending of PCL with lowmolecular weight poly-ethylene-oxide (PEO), which is biocompatible, water-soluble and hydrophobic in nature [13-15]. In previous research, PCL-PEO blends were created on lab-scale through a solution-based technique, producing a few grams of blended polymer at a time. It was found that for scaffolds manufactured from these blended materials; both the flexibility and surface hydrophilicity were significantly improved [1]. A next step, then, is to upscale the production process of the blends so that several hundreds of grams or even kilograms may be produced in a single run. Within the current research, a method was developed to compound the PCL and PEO fractions through double-screw extrusion, a melt-based process with the necessary output capacity. One of the foremost challenges to apply this technique is the apparent immiscibility of PCL and PEO in the melt phase [16]. Therefore, the production method was evaluated not just by absolute composition, but also based on an assessment of the effective distribution of the PCL and PEO phases within the polymer structure. Additionally, the effect of the blending method on the semicrystalline structure of the final polymer was quantified.

\section{Materials and Methods}

\section{Materials}

The PCL fraction used in this research was PCL Capa 6500 (Perstorp, UK) with a molecular weight of $50000 \mathrm{Da}$ and melt 
temperature of $58-60^{\circ} \mathrm{C}$. The PEO fraction (Ineos, Belgium) had a molecular weight of $10000 \mathrm{Da}$ and a melt temperature of $64^{\circ} \mathrm{C}$.

\section{Twin screw compounding}

The blends were compounded on a co-rotating twin screw extruder ZSK 18 MEGA-lab (Coperion, Belgium), with a progressive temperature profile of ( 9 sections, from intake to mould) 75-80-85-90-90-90-90-90-90 ${ }^{\circ} \mathrm{C}$. The machine is setup with a main and side feeder, each of them with a separate feed rate.

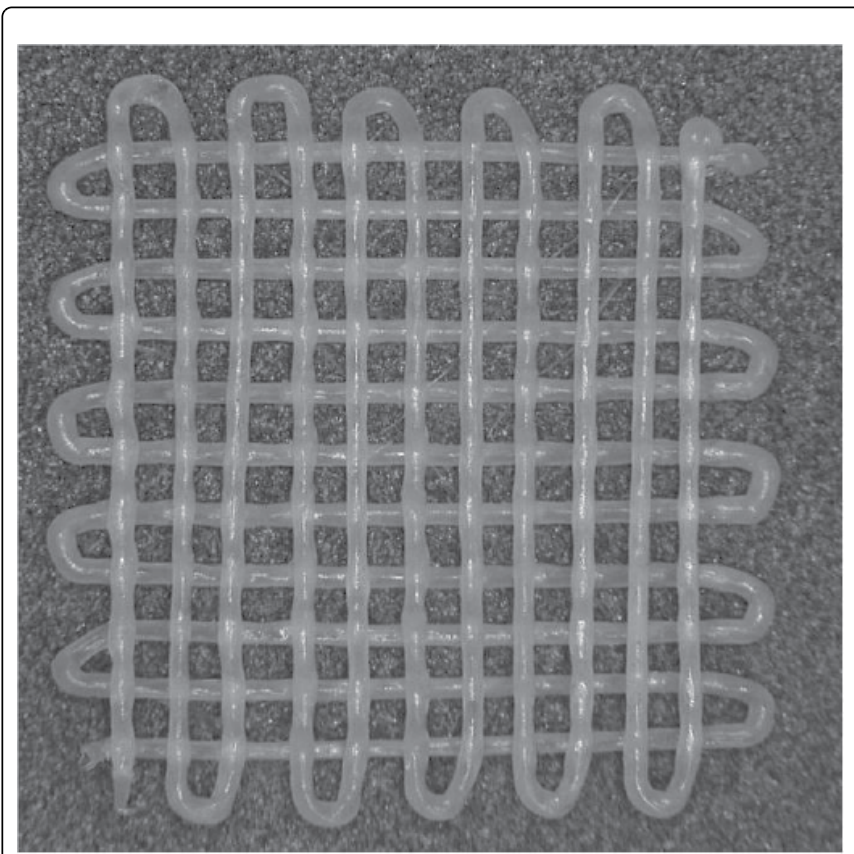

Figure 1: Example of a 3D plotted scaffold. Individual filaments are $180 \mu \mathrm{m}$ in diameter.

PCL was added via the main feeder and PEO via the side feeder, which is located towards the end of the compression zone. Feed rates were set to accomplish an 80/20 PCL/PEO blend. Extrusion screw speed was set at $300 \mathrm{rpm}$.

A filament die with wire diameter of $3 \mathrm{~mm}$ was used. Extruded wires were guided through a water bath for cooling after exiting the die.

Extruded wires were regranulated mechanically and the reground material was compounded anew (all via main feeder) with the same extrusion settings. This was repeated several times so as to assess the impact of multiple compounding steps on the distribution of PEO within the PCL. Characterization was performed for blends produced with one to five extrusion steps. These are labeled PCL-PEO-x, with $\mathrm{x}$ equal to the total number of extrusion steps.

\section{Blend composition}

The composition of the blends was verified through proton nuclear magnetic resonance (H-NMR), in which $10 \mathrm{mg}$ of blended polymer was dissolved in deuterated chloroform $(\mathrm{CDCl} 3)$ for the measurements. Tetra methylsilane (TMS) was used as an internal standard for calibrating the chemical shift. The experiments were performed on a WH $300 \mathrm{MHz}$ 1H-NMR apparatus (Bruker, Belgium) and were conducted in triplicate for PCL-PEO-1, -3 and -5 and compared to those of pure PCL and pure PEO. By integrating the PCL and $\mathrm{PEO}$ peaks the number of monomer units per polymer type is determined. Using each polymer's molecular mass, the degree of polymerization (DP) can be determined and from this the mass percentage of each fraction within the blend.

\section{Morphological analysis}

Pellets of the pure polymers were molten on a glass plate by heating to $80^{\circ} \mathrm{C}$ and gently compressed to a thin film. Subsequently, the specimens were removed from the hot plate and left to cool to the surrounding air. For the blended materials, thin slices $(15 \mu \mathrm{m})$ were taken from a molten lump, using a microtome (Leitz 1401) and fixed between 2 glass plates. These samples were evaluated by bright field (BFM), polarized light (PLM) and phase contrast (PCM) microscopy at different magnifications on a Olympus $\mathrm{BH} 2$ microscope, equipped with camera (Leica DFC 280) and image processing software (Leica Application Suite 4.0). Several slides, taken at different locations throughout the lump, were analyzed per blend sample.

The size of the spherulites was obtained using the small angle light scattering technique (SALS). The same slides with sections used for microscopy were used in the SALS technique. This system consists of an optical bench in which is mounted an HeNe laser of $0.8 \mathrm{~mm}$ diameter and with $632.8 \mathrm{~nm}$ wavelength, two crossed polarizers, a CCD camera and an analysis system Hamamatsu Hipic 6.3. Two measurements were made per specimen and at least three specimens were tested per blend sample.

\section{Results and Discussion}

\section{Blend compositions}

An example of the H-NMR spectra of the PCL-PEO-1 blend is shown in Figure 2, including the integration of the peaks. From the spectra of the pure materials, it was apparent that the peak at a chemical shift of around $3.6 \mathrm{ppm}$ is representative of PEO. This peak is attributed to the methylene protons of the $-\mathrm{CH} 2-\mathrm{CH} 2 \mathrm{O}$ - in the PEO chain structure [17]. The other peaks can be attributed to PCL, or in the case of the peak around $7.2 \mathrm{ppm}$, to both polymers.

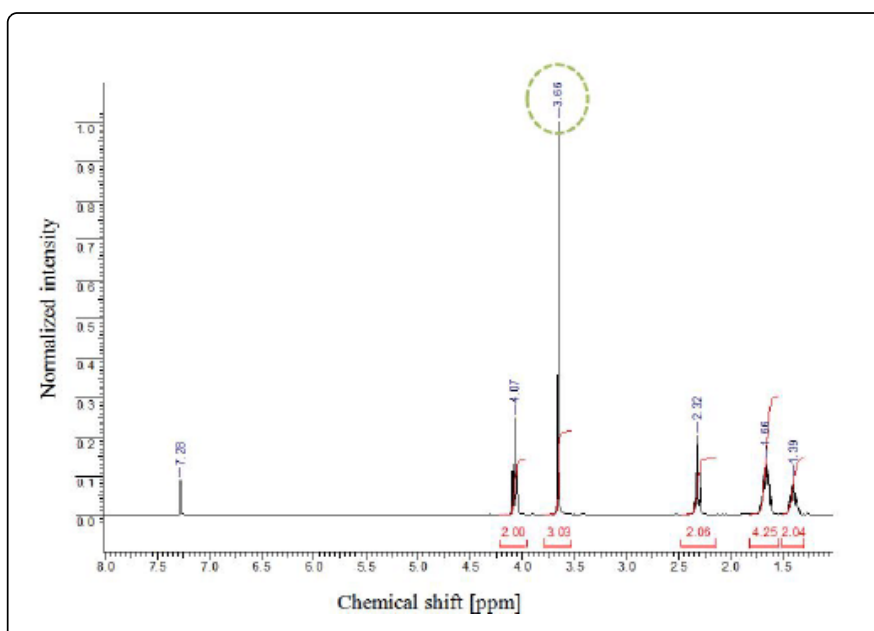

Figure 2: H-NMR spectrum of a PCL-PEO-1 sample. The peak with a chemical shift around $3.6 \mathrm{ppm}$ is indicative of PEO. 
The calculations of the weight percentage of PEO in the different blends from the H-NMR spectra yield the following results: $20.08 \pm 2.56$ $\%$ for PCL-PEO-1, 20.36 $\pm 0.12 \%$ for PCL-PEO-3 and $20.16 \pm 0.15 \%$ for PCL-PEO-5. Confirming an effective composition of 80/20 PCL-PEO, these results validate the effectiveness of the production method in terms of absolute composition of the blends. Regarding the distribution of the PEO within the PCL matrix, it is noted that the standard deviation on the results is remarkably smaller for the blends with 3 or more compounding steps, compared to the blend with only one extrusion step. As H-NMR is conducted on small amounts of material, this is a first indication of a more homogeneous distribution of PEO throughout the PCL for the blends with multiple extrusion steps. A more precise assessment of this distribution must be made via the morphological analysis.

\section{Morphological analysis}

Figure 3 shows PLM images of the crystalline structures of the unblended PCL and PEO, with a notably different magnification. PEO forms very large spherulites, with a few smaller spherulites appearing in the grain boundaries. Contrarily, PCL forms a relatively fine crystalline structure, in which the individual spherulites cannot be visualized at the used magnification of $1.67 \times 40$. When looking at the blended polymers in Figure 4, PLM in Figure 4a shows a structure similar to that of pure PCL, with an appearance of dark rounded regions, scattered throughout the PCL matrix. BFM of the same sample in Figure $4 \mathrm{~b}$ shows a clear phase separation between the matrix and the circular regions, indicating the immiscibility of the two phases. Examining the structure with greater magnification confirms the existence of circular phase boundaries in the BFM image of Figure 4c. Using PLM, it can be observed in Figure $4 \mathrm{~d}$ that the dispersed phase has a structure alike to that of PEO. Each island constitutes of a single large PEO spherulite. The well-rounded spherulitic form of these PEO zones indicates that upon cooling, the PEO crystals are first to nucleate and grow into the large spherulites, after which the PCL crystallites fill in the remaining space. Similar structures have previously been recorded for polyoxymethylene [18] and PCL-based [19] blends incorporating PEO.
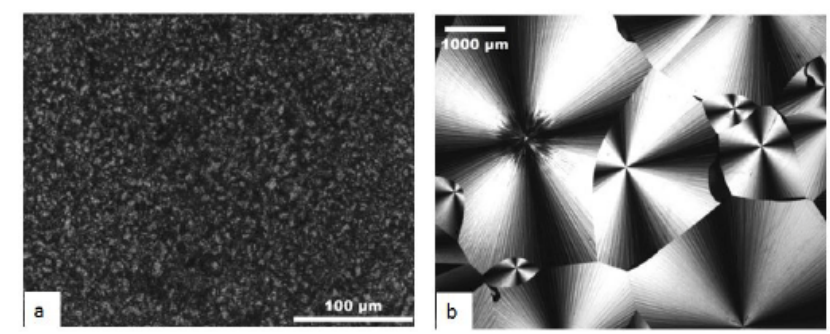

Figure 3: (a) PLM images of the semi-crystalline structures of (a) pure PCL, magnification 40x1.67 and (b) pure PEO, magnification $2 \times 1.67$.

To assess the distribution of the PEO throughout the PCL matrix, PCM is used. Contrary to PLM, PCM does not allow discerning the specific semi crystalline structure of the polymer components, but it does result in a clearer distinction between the two phases, as is shown in Figure 5a. For the PCL-PEO-1 slices, sizeable areas are observed which appear darker in the PCM images (Figure 5b). When inspecting these areas with higher magnifications (Figure 5c), it becomes evident that these regions do not contain any dispersed PEO nodules. Such PEO-poor zones were found in nearly all PCL-PEO-1 samples, excluding the possibility of a random occurrence. These zones indicate that a fully homogeneous blending of PEO into PCL did not occur after the first extrusion step. Examination of PCL-PEO-2 showed that such PEO-poor zones did remain to some degree, but that they were much smaller and scarcer (on average, about 20X smaller). There was no significant further evolution in the size or occurrence of these zones for slices from PCL-PEO-3 to -5. An example of such a zone found in PCL-PEO-5 is shown in Figure 5d.

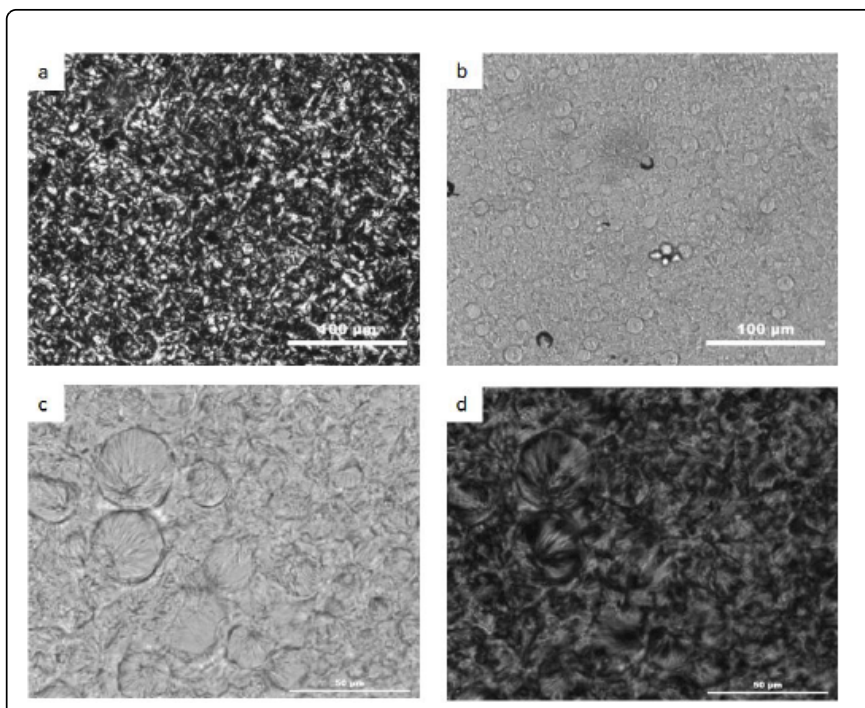

Figure 4: Structure of the blended polymers: (a) PLM image of PCL-PEO-1 (40x1.67) and (b) BFM image of the same region. (c) BFM image of PCL-PEO-5 (40x3.3) and (d) PLM image of the same region.

Concerning the SALS results, average crystallite sizes (PCL and PEO together) were found to be $13.85 \pm 1.62 \mu \mathrm{m}$ for PCL-PEO-1, $7.89 \pm 0.38 \mu \mathrm{m}$ for PCL-PEO-2, $8.09 \pm 0.52 \mu \mathrm{m}$ for PCL-PEO-3, $8.01 \pm 0.15 \mu \mathrm{m}$ for PCL-PEO-4 and $7.93 \pm 0.73 \mu \mathrm{m}$ for PCL-PEO-5. The spherulites are clearly larger for PCL-PEO-1, while no significant difference is observed between the samples with multiple extrusion steps. Smaller spherulites indicate a larger degree of nucleation during the crystallization of the melt. If the PEO is better dispersed within the melt of PCL, the already crystallized PEO will better serve as a site of heterogeneous nucleation for the PCL, thus raising PCL's nucleation rate.

Both the microscopic analysis and the SALS results lead to understand that the blending of the PEO into the PCL matrix is notably more homogeneous when extruding the blend a second time, while any further compounding steps do not significantly improve the dispersion of the PEO.

During the initial extrusion step, the PEO is only added (in solid form) to the PCL melt at the end of the compression zone. As such, the residence time of the $\mathrm{PEO}$ is insufficient for it to be kneaded homogeneously into the PCL melt matrix by the twin screws during this first step. During the second extrusion, however, the PCL and PEO fractions are already mixed within the reground wires and are brought into the melt together in the intake section of the screws. This results in a far more homogeneous distribution of PEO within the 
blend. Small PEO-poor zones continue to exist, but these are considered acceptable in size and occurrence.

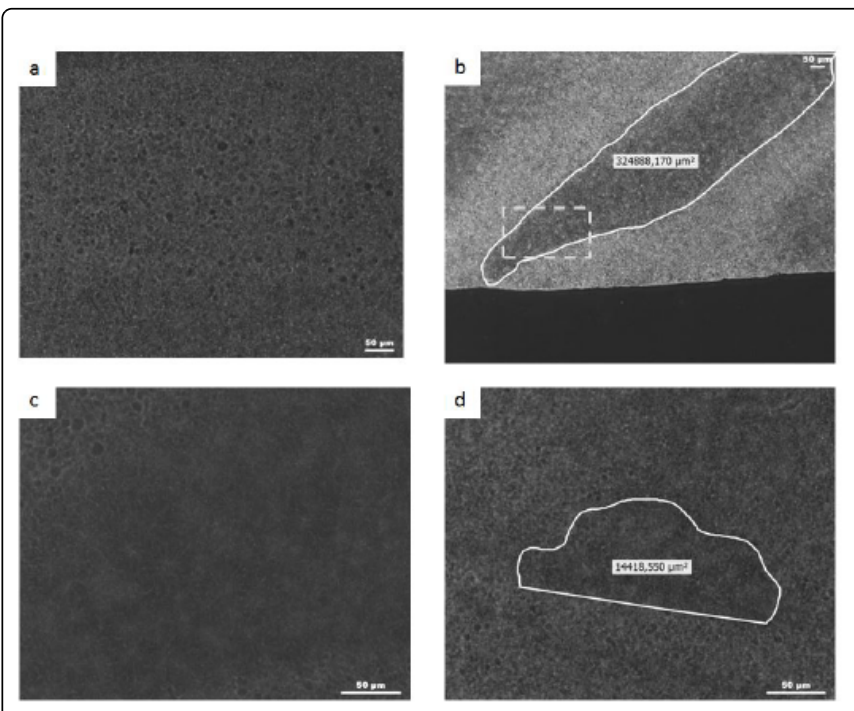

Figure 5: PCM images of the blends: (a) typical structure of a wellblended region example, (b) large PEO-poor region in PCL-PEO-1 sample, (c) enlargement of indicated area in (b) and (d) smaller PEO-poor region in PCL-PEO-5 sample.

\section{Conclusions}

In this research, it was found that by regrinding and re-extruding twin-screw compounded blends of PCL/PEO, it is possible to obtain sufficiently homogenized dispersions of the PEO spherulites within the PCL matrix. Small PEO-poor zones continue to exist, but these are considered acceptable in size and occurrence.

Further compounding steps were found to have no significant effect on the blend's structural properties. Given the polymers' inherent immiscibility in one another, phase separation between the PCL and PEO will continue to occur as long as the materials are not chemically copolymerized. However, the current method makes it possible to produce $\mathrm{PCL} / \mathrm{PEO}$ blends in bulk, producing several kilograms at a time, using a straightforward and easy method.

\section{Acknowledgements}

The authors would like to acknowledge Prof. Peter Dubruel, Dr. Sandra Van Vlierberghe, Mrs. Olga Musial and Mr. Geert-Jan Graulus from the PBM (Polymer Chemistry \& Biomaterials) Group at Ghent University, for their help with the NMR tests and the delivery of the PEO material. Likewise, the authors want to thank Mr. Mauricio Malheiro for his assistance with the microscopy.

\section{References}

1. Ragaert K, De Somer F, Van de Velde S, Degrieck J, Cardon L (2013) Methods for Improved Flexural Mechanical Properties of 3D-Plotted PCL-
Based Scaffolds for Heart Valve Tissue Engineering. Journal of Mechanical Engineering 59: 669-676.

2. Brody S, Pandit A (2007) Approaches to heart valve tissue engineering scaffold design. J Biomed Mater Res B Appl Biomater 83: 16-43.

3. Sivalingam G, Madras G (2003) Thermal degradation of poly $(\varepsilon-$ caprolactone). Polymer Degradation and Stability 80: 11-16.

4. Eshraghi S, Das S (2010) Mechanical and microstructural properties of polycaprolactone scaffolds with one-dimensional, two-dimensional, and three-dimensional orthogonally oriented porous architectures produced by selective laser sintering. Acta Biomater 6: 2467-2476.

5. Guarino V1, Causa F, Taddei P, di Foggia M, Ciapetti G, et al. (2008) Polylactic acid fibre-reinforced polycaprolactone scaffolds for bone tissue engineering. Biomaterials 29: 3662-3670.

6. Wang ZW, Su JC, Ma YH, Zhang X, Cao LH, et al. (2010) Preparation and Properties of Nano Calcium Deficient Apatite/Poly ( $\varepsilon$-caprolactone) Composite Scaffold. Journal of Inorganic Materials 25: 500-506.

7. Lam CX, Hutmacher DW, Schantz JT, Woodruff MA, Teoh SH (2009) Evaluation of polycaprolactone scaffold degradation for 6 months in vitro and in vivo. J Biomed Mater Res A 90: 906-919.

8. Nair LS, Laurencin CT (2007) Biodegradable polymers as biomaterials. Progress in Polymer Science 32: 762-798.

9. Lee H, Kim G (2010) Biocomposites electrospun with poly( $\varepsilon$-caprolactone) and silk fibroin powder for biomedical applications. J Biomater Sci Polym Ed 21: 1687-1699.

10. Shan X, Yuan Y, Liu C, Tao X, Sheng Y, et al. (2009) Influence of PEG chain on the complement activation suppression and longevity in vivo prolongation of the PCL biomedical nanoparticles. Biomed Microdevices 11: 1187-1194.

11. Yao K, Wang J, Zhang W, Lee JS, Wang C, et al. (2011) Degradable rosinester-caprolactone graft copolymers. Biomacromolecules 12: 2171-2177.

12. Desmet T, Billiet T, Berneel E, Cornelissen R, Schaubroeck D, et al. (2010) Post-plasma grafting of AEMA as a versatile tool to biofunctionalise polyesters for tissue engineering. Macromol Biosci 10: 1484-1494.

13. Kim G, Park J, Park S (2007) Surface-treated and multilayered poly( $(\varepsilon-$ caprolactone) nanofiber webs exhibiting enhanced hydrophilicity. J Polym Sci B Polym Phys 45: 2038-2045.

14. Guarino V, Netti PA, Ambrosio L (2008) Development of Highly Oriented Porous Structures by PCL/PEO Co-Continuous Blends. Proceedings of 4 th International Conference Times of Polymers (TOP) and Composites, Ischia, Italy.

15. Reignier J, Huneault MA (2006) Preparation of interconnected poly( $\varepsilon$ caprolactone) porous scaffolds by a combination of polymer and salt particulate leaching. Polymer 47: 4703-4717.

16. Qiu Z, Ikehara T, Nishi T (2003) Miscibility and crystallization of poly(ethylene oxide) and poly(e-caprolactone) blends. Polymer 44: 3101-3106.

17. Ma G, Miao B, Song C (2010) Thermosensitive PCL-PEG-PCL hydrogels: Synthesis, characterization, and delivery of proteins. J Appl Polym Sci 116: 1985-1993.

18. Gao Y, Sun S, He Y, Wang X, Wu D (2011) Effect of poly(ethylene oxide) on tribological performance and impact fracture behavior of polyoxymethylene/polytetrafluoroethylene fiber composites. Composites Part B: Engineering 42: 1945-1955.

19. Chuang WT, Jeng US, Sheu HS, Hong PD (2006) Competition between phase separation and crystallization in a PCL/PEG polymer blend captured by synchronized SAXS, WAXS, and DSC. Macromolecular Research 14: 45-51. 